\title{
LA FRAgilidad de los CONSENSOS. Polarización ideOlógica en el Chile post Pinochet
}

\author{
The Fragility of Consensus: Ideological Polarization \\ in Post-Pinochet Chile
}

\section{JAIME LINDH}

IdeaPaís, Chile

\author{
JORGE FÁBREGA \\ Universidad del Desarrollo, Chile
}

\section{JORGE GONZÁLEZ}

Universidad Adolfo Ibáñez, Chile

\begin{abstract}
RESUMEN
El artículo presenta evidencia de la polarización ideológica a nivel político, socioeconómico y generacional en la población chilena entre 1990 y 2017. Utilizando datos de la Encuesta de Opinión Pública del Centro de Estudios Públicos (CEP), se estima indirectamente la ideología de los individuos con la metodología propuesta por Poole (1998). Los principales hallazgos son: (i) en 1993, adherentes y opositores al gobierno de turno inician un proceso de distanciamiento ideológico que se consolida a mediados de 2008; (ii) la distancia ideológica entre los niveles socioeconómicos alto y bajo empieza a aumentar desde 2005 en adelante; y, finalmente, (iii) a partir de 2011, la generación nacida antes de 1955 empieza a alejarse ideológicamente de aquellos nacidos después de 1980.
\end{abstract}

Palabras clave: polarización, opinión pública, ideología, Chile, teoría espacial del voto

\begin{abstract}
This article presents evidence of political, socioeconomic and generational polarization among the Chilean population between 1990 and 2017. Using data from the Public Opinion Survey of the CEP, the ideology of the individuals is estimated indirectly using the methodology developed by Poole (1998). The main findings are the following: (i) in 1993, adherents and opponents of the current government initiated a process of ideological distancing that was consolidated in mid-2008; (ii) the ideological distance between high and low socioeconomic levels began to increase from 2005 onwards; and finally, (iii) the generation born before 1955 began to distance itself ideologically from the one born after 1980, since 2011.
\end{abstract}

Keywords: polarization, public opinion, ideology, Chile, spatial theory of voting. 


\section{INTRODUCCIÓN}

Si bien la búsqueda de consensos ha caracterizado al Chile post Pinochet, finalizando la segunda década del presente siglo, comentarios y análisis consistentes con una mayor polarización han puesto en duda su real alcance en el tiempo. Por ejemplo, en marzo de 2018, la historiadora Lucía Santa Cruz afirmó que el país se había polarizado con el segundo gobierno de la presidenta Michelle Bachelet (García 2018). Asimismo, en agosto de 2017, el periodista y cientista político Mauricio Morales argumentó que — con el nuevo voto voluntario y en un contexto de apatía hacia la política- los candidatos presidenciales estarían enfrentando incentivos a movilizar al electorado con ideas más polarizadas (Morales 2017). Sin embargo, hay otros que han sugerido lo contrario. Entre estas voces destaca la del economista Harald Beyer, quien mencionara que "... más allá de la crispación que hay en la elite, la población no está polarizada" (Valenzuela y Muñoz 2017).

No obstante, la discusión sobre si existe o no mayor polarización ha carecido de una revisión sistemática y de largo plazo que permita ponderar los argumentos expuestos en el debate público. Este artículo busca contribuir a esta discusión estudiando este fenómeno en la población chilena durante el periodo 19902017. La polarización se define como un proceso en que las preferencias de los individuos se van concentrando en campos opuestos.

Si bien no está resuelto que toda polarización sea perjudicial, en el caso chileno hay un sesgo importante en contra de ella, proveniente de la traumática experiencia vivida entre 1973 y 1990. En dicha época, Chile llegó a niveles de desencuentro tan profundos como a los que apuntaron Constable y Valenzuela (1993) en su análisis del régimen de Pinochet al titularlo “Una nación de enemigos. Chile bajo Pinochet".

De ahí que la polarización no es ajena al debate público chileno. Es más, la discusión respecto a su existencia no es del todo nueva, sino que se venía intensificado desde - al menos- las masivas movilizaciones sociales durante la primera administración de Bachelet (2006-2010). De hecho, en octubre de 2007, el presidente de la Cámara de Diputados, Patricio Walker, llamó a "restaurar la política de los consensos" (Agencia ORBE 2007). Luego, en octubre de 2011, en el apogeo de las movilizaciones sociales, el diputado Jorge Tarud señaló que en el país había "una polarización sobre todo en el ámbito político" (Agencia UPI 2011). El asunto se posicionó a tal nivel que, en mayo de 2014, un importante periódico del país publicó en su editorial que "el progreso ha traído como una consecuencia paradójica una mayor polarización política" (El Mercurio 2014).

En este contexto, se estudiará la existencia o no de polarización en la población general de Chile y en diversos grupos de ésta, construidos en base a atributos políticos, socioeconómicos y generacionales. La justificación de la elección de dichos grupos radica en que, durante los 27 años posteriores al retorno a la democracia, la sociedad chilena experimentó profundos cambios. Uno de 
los más relevante fue el contraste entre la época determinada por el quiebre democrático en 1973 y la exitosa transición, caracterizada por niveles de cooperación sin precedentes entre la Concertación y la coalición opositora. Por lo tanto, el cambio desde un contexto dividido hacia uno más armonioso explica la importancia de estudiar la polarización en cuanto a las posiciones políticas que los chilenos fueron manifestando durante este tiempo. Las posiciones políticas de los habitantes de este país se identifican a través de los apoyos que fueron declarando a los gobiernos de turno.

Además, en este periodo, Chile se caracterizó por un alto crecimiento económico, siendo hoy el país de mayor ingreso per cápita en Latinoamérica (Fondo Monetario Internacional 2017). Lo anterior, sumado a la extensión del acceso a la educación secundaria y terciaria, indica que los chilenos vivieron importantes cambios en sus condiciones materiales, lo cual vuelve relevante el escrutinio de la polarización a nivel socioeconómico. Los avances en superación de la pobreza y el surgimiento de nuevas clases medias conllevan a la necesidad de identificar los cambios políticos que dichos grupos vivieron durante estas tres décadas.

Finalmente, la transición a la democracia y el crecimiento económico implicó el surgimiento de importantes brechas generacionales. Por un lado, hubo avances sustantivos en materias de transparencia y rendición de cuentas, los que unidos a los cambios tecnológicos- incidieron en un aumento en los niveles de información adquirida por las nuevas generaciones. Asimismo, fueron los más jóvenes los principales beneficiarios del desarrollo, en cuanto a menor desigualdad, mayor movilidad y menor pobreza (Sapelli 2014). Por otro lado, las experiencias de vida de aquellos en quienes influyó la estridencia a la que se refirieron Constable y Valenzuela (1993), contrasta con la experiencia de cooperación experimentada posteriormente. Esto conlleva a que sea igualmente pertinente realizar un análisis de polarización a nivel generacional.

El artículo examina la población chilena utilizando los datos de la Encuesta de Opinión Pública del Centro de Estudios Públicos (CEP). Se propone estudiar la polarización desde un enfoque ideológico, es decir, observando los cambios que experimenta la distribución ideológica de los chilenos. La ideología se estima indirectamente a través de la propuesta metodológica de Poole (1998). Esta decisión contrasta con el enfoque temático, aproximación predominante en la literatura, que analiza la distribución de opinión de los individuos respecto a temas públicos, tales como el aborto y el matrimonio homosexual, entre otros. Dado que las ideologías configuran la interacción política entre votantes y actores políticos (Hinich y Munger 1994; Poole y Rosenthal 1997), es preferible usar el enfoque ideológico respecto al temático. Esto se sustenta en el hecho de que un escenario con individuos ideológicamente polarizados conllevaría a que los actores políticos tuvieran mayores incentivos para formular políticas públicas divergentes. En contraste, la existencia de polarización temática es condición necesaria - pero no suficiente- para incentivar la divergencia programática entre los políticos, toda vez que el tema debe ser necesariamente saliente en 
la competencia política para provocar disenso. Adicionalmente, para el caso específico de Chile, es conveniente usar un enfoque ideológico debido a que la centralidad de la figura de Pinochet minimizó el efecto político en la capacidad de consensuar de otras diferencias temáticas durante una parte significativa del periodo posterior a 1990 .

El trabajo se ordena de la siguiente forma: el apartado II discute sobre la polarización, seguido del apartado III que describe la metodología y datos. El apartado IV muestra los resultados finalizando con el apartado V que exhibe las conclusiones.

\section{REVISIÓN DE LA LITERATURA}

\section{¿Cómo entender la polarización?}

Sartori (2005), en su tipología de sistema de partidos, sostiene que "tenemos polarización cuando tenemos distancia ideológica" (2005, 120). Entre los sistemas que describe el autor, el pluralismo polarizado se caracteriza, entre otros aspectos, por estar fragmentado en varios partidos que están distanciados unos de otros en términos ideológicos. Dalton (2008), en su planteamiento respecto a que los sistemas deben evaluarse no solo por su fragmentación, sino que también por su polarización, afirma que "... un sistema con un gran número de partidos en los extremos políticos, es un sistema polarizado..." (2008, 903). Entonces, en términos de Cox (1990), se podría plantear que un sistema polarizado es aquel en donde las fuerzas centrífugas dominan a las fuerzas centrípetas, entendiendo por las primeras a aquellas que llevan al distanciamiento ideológico entre diferentes partidos políticos y las segundas al efecto contrario.

El estudio de la polarización en las élites políticas también ha sido un tema ampliamente tratado. Poole y Rosenthal (1984) muestran que el Senado de EE. UU. se polarizó desde mediados de la década de 1970, evidenciando un movimiento de los actores desde el centro hacia los extremos. Asimismo, Brady y Han (2004) señalan que, en el pasado, la élite política americana ya había estado polarizada, mostrando brechas importantes entre los partidos políticos. En tanto McCarty et al. (2006) postulan que este fenómeno se caracteriza por dos cosas: "primero (...) los moderados van desapareciendo. Segundo, los dos partidos se apartan" (2006, 3). Por lo tanto, la polarización no solo se plantea como un distanciamiento hacia los extremos, sino también como un detrimento de los moderados.

La evidente polarización entre los actores políticos motivó, en la última década del siglo $\mathrm{XX}$, que este fenómeno se estudiara en la opinión pública estadounidense. Sin embargo, las investigaciones no han llegado a resultados concluyentes, existiendo quienes sugieren polarización (Hetherington 2008; Layman et al. 2006; Abramowitz y Saunders 2008) y otros lo contrario (Di Maggio 
et al. 1996; Evans 2003; Fiorina et al. 2005). Según Lee (2015), las diversas formas de entender el concepto explicarían este hecho. De esta discusión hay al menos dos planteamientos que resaltan. El primero propone que la polarización es un proceso que mueve a los individuos hacia posiciones extremas, mientras que el segundo sugiere que la polarización es un proceso en que se forman grupos de personas con preferencias opuestas e incompatibles. El primer punto —que está presente en la literatura a nivel de élites - lo ilustran Fiorina y Abrams (2008), sosteniendo que "el movimiento lejos del centro, hacia los extremos, pareciera ser una definición no controvertida de polarización" $(2008,567)$. Mientras que el segundo aspecto es descrito por Esteban y Ray (1994) al postular que una sociedad está polarizada si “la población se agrupa en clusters significativamente grandes, tal que cada cluster sea muy similar en términos de los atributos de sus integrantes, pero diferentes clusters tengan integrantes con atributos muy distintos" (1994, 819). En Di Maggio et al. (1996), ambos elementos están presentes cuando mencionan que la polarización "(...) atenta contra la estabilidad social y política, reduciendo la probabilidad de que se formen grupos al centro de la distribución de opinión y aumentando la posibilidad de que se formen grupos con preferencias políticas distintas e irreconciliables" $(1996,693)$.

En consecuencia, la polarización es un concepto estudiado a nivel de sistemas de partidos, en élites políticas y como opinión pública. Una definición transversal para estos tres campos estipularía que la polarización es un proceso en que los actores políticos o las preferencias individuales se van concentrando en polos opuestos. En el artículo se utilizará esta definición para medir su presencia en la población general de Chile y en diversos grupos al interior de esta, de modo tal de identificar si se han desplazado hacia extremos opuestos o no.

\section{¿En qué tema importa la polarización?}

Son múltiples los asuntos en que los individuos pueden polarizarse, tales como en temáticas económicas, valóricas o culturales. Esto conlleva a la necesidad de definir en qué temas sería pertinente el estudio del fenómeno.

Al menos en el caso chileno, la relevancia de la polarización —como objeto de estudio - radica en sus efectos y, en particular, en el deterioro de la posibilidad de convergencia entre los actores políticos. Por lo tanto, la polarización será relevante en la medida que incentive a los actores políticos a representar a los extremos en desmedro del centro. El desafío - en consecuencia- está en identificar las temáticas que configuran la relación entre votantes y actores políticos.

A partir del trabajo seminal de Downs (1957), la Teoría Espacial del Voto (TEV) entrega un marco teórico adecuado para el estudio de la polarización, comprendiendo la competencia política como un mercado entre votantes y políticos, que transan votos por promesas de políticas públicas. En su estado actual de desarrollo, la TEV es un conjunto de técnicas usadas para develar las 
preferencias reveladas de individuos (sean políticos o ciudadanos) a partir de sus acciones (votos, expresiones escritas, opiniones o evaluaciones) bajo la consideración de cualquier marco de interacción (reglas e instituciones) que los vinculen. No obstante, debido a la gran influencia que tuvo el trabajo de Downs y, en particular, su teorema del votante medio, por mucho tiempo se consideró a la TEV como una herramienta conceptual cuyo uso exclusivo era estudiar votaciones en sistemas políticos mayoritarios con solo dos candidatos. Si bien ya en Hinich (1977) se muestra que la TEV no requiere ni descansa en los resultados del votante mediano para analizar la competencia política en diversos escenarios institucionales y electorales, no es menos cierto que el análisis basado en TEV se limitó por mucho tiempo al estudio del caso mayoritario en el contexto norteamericano. Recién en las últimas décadas, estudios basados en TEV aplicados en otros países comienzan a ser recurrentes en las revistas académicas. Por ejemplo, en Francia (Rosenthal y Voeten 2004), Turquía (Çarkoğlu y Hinich 2006) y Latinoamérica (León 2013; Saiegh 2014). En el caso chileno, destacan los estudios de Dow (1998), Magar et al. (1998) y Bonilla (2002).

En cuanto a la competencia política, si bien en un inicio la TEV consideraba que ciertos temas públicos configuraban la relación entre votantes y políticos, Hinich y Munger (1994) demuestran que —independiente de cuales sean estos asuntos - hay dimensiones latentes que determinan dicha interacción, las cuales denominan "ideologías". Posteriormente, Poole y Rosenthal (1997) refuerzan esta conceptualización al evidenciar que las votaciones en el Congreso de EE. UU., a lo largo de su historia, se podían configurar en un eje unidimensional ideológico liberalismo-conservadurismo.

De este modo, la TEV vincula las preferencias políticas del votante con las de los actores políticos. Esto implica que las personas votarán por el político que perciban ideológicamente más cercano. Además, asume que el político maximiza su cuota de poder posicionándose en el segmento ideológico que entregue una mayor posibilidad de triunfo. Por lo tanto, la TEV plantea que el político expresa promesas de políticas públicas como una estrategia de posicionamiento en dicho segmento.

En consecuencia, a mayor similitud ideológica entre las personas, más probable será el consenso entre las propuestas de política pública de los políticos. En cambio, en un escenario con individuos ideológicamente polarizados, menor será dicha posibilidad.

En consideración a lo anterior, la polarización se estudia desde un enfoque ideológico, el cual permite superar ciertas trabas asociadas al enfoque temático, como es la aproximación usada principalmente en la literatura. El enfoque temático busca rasgos de polarización en las opiniones que tienen los individuos respecto a diversos temas públicos. Un problema común en este enfoque es que se deben definir umbrales de temas que, una vez superados, darían paso a la polarización. Por ejemplo, Di Maggio et al. (1996) y Evans (2003) concluyen que en EE. UU. hay "una leve polarización" al encontrar evidencia solo respecto 
al aborto y la sexualidad. Sin embargo, ¿cuál es el criterio para sostener que la polarización en estos temas no provocará disputas?

La presencia de polarización en un tema público no genera conflicto si el asunto no es saliente en las preferencias electorales de los individuos. Es decir, la polarización en un tema público es condición necesaria pero no suficiente para generar disensos, dado que el elemento polarizado no implicará necesariamente desacuerdo entre los actores políticos. El siguiente ejemplo ilustra este punto: en un año $n$ los actores $j$ y $k$ utilizan el tema $x$ para posicionarse en sus respectivos segmentos objetivos. No obstante, en el año $n+1$ el tema que marca la discusión pública es $z$ que, a diferencia de $x$, no se polarizó. El enfoque temático buscará polarización en la distribución de opinión respecto a los temas $x$ y z. Luego, el enfoque temático concluirá que hay polarización, ya que existe en $x$. Sin embargo, la presencia de polarización en $x$ es relevante si y solo si en el año $n+1$ sigue siendo $x$ un tema saliente. Si fuera este el caso, los políticos $j$ y $k$ tendrían los incentivos para formular políticas divergentes basadas en $x$. Pero, en $n+1$, el tema saliente es $z$, que no presenta polarización. Entonces, en $n+1$ los actores políticos no tienen incentivos divergentes. La disputa respecto a legalización del aborto al interior de la Concertación ejemplifica este asunto, ya que no alcanzó a tener un efecto que impidiera el consenso durante los 20 años en que dicha coalición se mantuvo en el poder. En contraste, el enfoque ideológico analizará la distribución ideológica de los individuos. De esta forma, observa directamente la evolución de la dimensión explicativa de la conducta electoral de los políticos, independiente de los temas públicos que están siendo discutidos en una elección.

Se han planteado formas alternativas para identificar polarización en la opinión pública más allá del enfoque temático. Primero, se postuló la correlación entre las opiniones que tienen los individuos en diversos temas públicos (Gelman 2008; Baldassarri y Gelman 2008), segundo, la relación entre las opiniones de las personas y su pertenencia partidista (Fiorina et al. 2005; Fiorina y Abrams 2008; Gelman 2008; Baldssarri y Gelman 2008; Levendusky 2009) y, tercero, la relación entre la pertenencia ideológica y partidista (Abramowitz y Saunders 2008; Gelman 2008). Si bien estas alternativas permiten identificar el peso de la ideología o del partidismo en la formación de preferencias, no capturan los cambios - acercamientos o distanciamientos - que experimentan los individuos en términos ideológicos.

En esta última dirección hay estudios que utilizan preguntas de autoclasificación ideológica (Abramowitz y Saunders 2006, 2008; Abramowitz 2010; Fiorina et al. 2005; Fiorina y Abrams 2008). Sin embargo, estas son problemáticas porque la interpretación de quien responde tienen un sesgo asociado a la escala. Por ejemplo, dos personas que discrepen ideológicamente podrían autoclasificarse como personas de centro, pese a sus diferencias.

En contraposición, la TEV dispone de metodologías que estiman indirectamente la posición ideológica de los individuos en función de las respuestas que 
entregan a ciertos estímulos (Enelow y Hinich 1984; Hinich y Munger 1994; Poole 1998). En esta línea, destacan los trabajos de Lee (2015) y Saiegh (2014), los que utilizan métodos de la TEv para estudiar la polarización de masas. Por lo tanto, para analizar si existe polarización en Chile durante 1990-2017, se requiere de una serie de datos que estén disponibles en dicho periodo y que contengan un mismo conjunto de preguntas de estímulo. Al respecto, las encuestas del CEP representan la única alternativa rigurosa y disponible. Además, en su interior, la pregunta más constante es la de evaluación a actores políticos, que se utilizará como estímulo, al ser consistente con la teoría.

\section{METODOLOGÍA Y DATOS}

\section{¿Cómo medir la polarización?}

Para medir la polarización en la opinión pública chilena, el artículo utiliza dos aproximaciones metodológicas —basándose en Di Maggio et al. (1996) denominadas polarización de la población y polarización entre grupos.

La primera analiza la población general del país. En particular, si existe o no un proceso en que la ideología de los chilenos haya empezado a concentrase en los extremos, reflejándose esto en una distribución cada vez más bimodal y de centro vacío.

Esta identificación está presente en múltiples artículos de polarización a nivel de opinión pública. Esteban y Ray (1994) demuestran que ella se refleja en una distribución bimodal alejada del centro. Hegselmann y Krause (2002) también consideran esta concepción cuando desarrollan modelos que explican el efecto de la confianza sobre la formación de opinión, destacando tres posibles escenarios en materia de distribución: la fragmentación (multimodalidad), la polarización (bimodalidad) y el consenso (unimodalidad). En tanto, Fiorina et at. (2005) escudriñan la opinión pública estadounidense considerando que un escenario polarizado será uno distribuido bimodalmente. Baldassarri y Bearman (2007) también conciben la polarización como un proceso en que se pierde densidad en el centro.

Los indicadores más usados para medir la polarización de la población son la varianza y la kurtosis (Di Maggio et al. 1996; Evans 2003; Baldassarri y Bearman 2007). Formalmente, se mide la varianza de la ideología $I$ en una sociedad compuesta por $n$ individuos en un año $t$ :

$$
S_{t}^{2}=\frac{\sum\left(I_{i t}-\bar{I}_{t}\right)^{2}}{n_{t}-1}
$$


En tanto, la kurtosis se obtiene a través de:

$$
k_{t}=\left[\frac{\frac{\sum\left(I_{i t}-I_{t}\right)^{4}}{n_{t}}}{s_{t}}\right]-3
$$

Para ilustrar la relación entre estos indicadores y la polarización de la población, la Figura 1 presenta cuatro tipos de distribuciones.

En el Gráfico 1, la ideología se concentra en la mediana, siendo evidente el consenso. Esta sociedad sin polarización se caracteriza por una baja dispersión (var $=0,4)$ y una alta modalidad $(\mathrm{k}=1,8)$. El Gráfico 2 presenta una sociedad distribuida nuevamente sobre un punto mediano, pero sin el nivel de consenso anterior. En contraste al Gráfico 1, acá hay más dispersión (var $=0,8$ ) y menos modalidad $(k=-0,4)$. En el Gráfico 3 ya no existe un acuerdo único, sino que hay dos puntos diferentes pero cercanos entre sí. Respecto al primer caso, la ideología es más dispersa $(\mathrm{var}=0,6)$ y menos modal $(\mathrm{k}=-1,9)$. Finalmente, el Gráfico 4 muestra nuevamente dos puntos distintos, pero esta vez distantes uno de otro. Entre las cuatro sociedades, la más polarizada es la última, siendo la que tiene la mayor dispersión (var $=6,4)$ y menor modalidad $(k=-2)$. En conclusión, habrá mayor polarización de la población en la medida que la distribución ideológica de los individuos sea más dispersa y menos modal. 
Figura 1. Relación entre varianza/kurtosis y polarización

Gráfico 3: Distribución bimodal y con dispersión

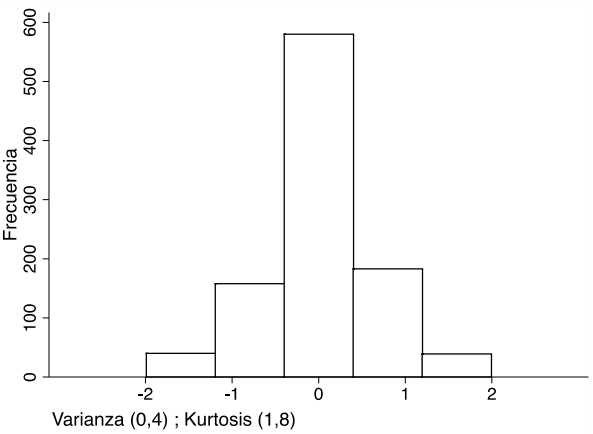

Gráfico 3: Distribución bimodal y con dispersión

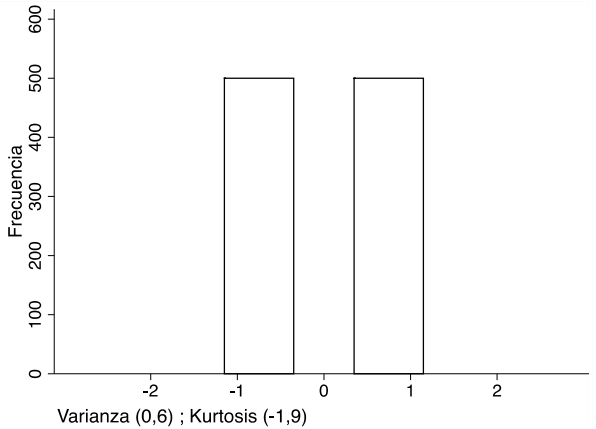

Gráfico 2: Distribución unimodal con mayor dispersión

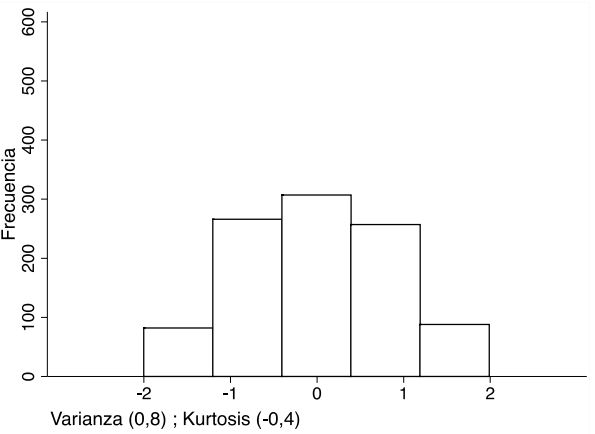

Gráfico 4: Distribución bimodal con mayor dispersión

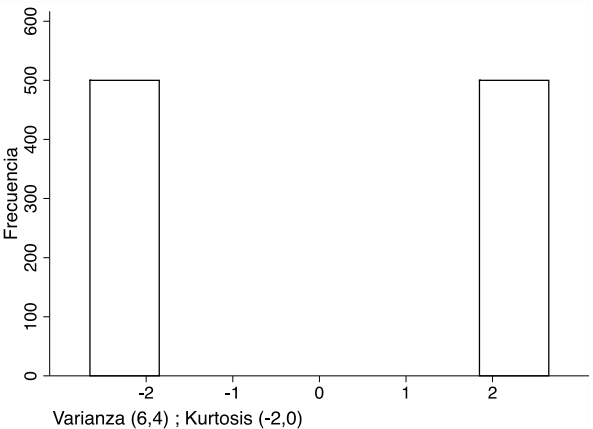

Fuente: Elaboración propia

Por otra parte, la segunda aproximación - la polarización entre grupos- busca medir la polarización en diversos grupos de la población. En particular, si se están formando o no grupos distantes unos de otros y con preferencias incompatibles.

Esta conceptualización está presente en diversos autores (Di Maggio et al. 1996; Abramowitz y Saunders 2006, 2008; Baldassarri y Bearman 2007; Gelman 2008). Destaca el trabajo de Di Maggio et al. (1996) por usar la diferencia de medias al evaluar qué tan distinta es la opinión entre dos grupos (heterogeneidad entregrupo) y la kurtosis, a fin de identificar qué tan cohesionada es la opinión al interior de cada uno (homogeneidad intra-grupo). Estos autores plantean que mientras mayor sea la heterogeneidad entre-grupo y mayor sea la homogeneidad intra, mayor polarización habrá.

Considerando lo anterior, la polarización entre grupos se mide a través de dos indicadores. Primero, en cuanto a la heterogeneidad entre-grupos, por medio de 
la diferencia de media. Formalmente, en un año $t$, la diferencia en la ideología $I$ entre un grupo $p$ y $q$ se mide a través de:

$$
d_{t}^{J}=\bar{I}_{t}^{p}-\overline{I_{t}^{q}}, \text { siendo } p, q \subseteq J ; p \neq q
$$

Segundo, en cuanto a la homogeneidad intra-grupo, se utiliza la kurtosis:

$$
k_{t}^{p}=\left[\frac{\frac{\sum\left(I_{i t}^{p}-\overline{I_{t}^{p}}\right)^{4}}{n_{t}^{p}}}{s_{t}^{p}}\right]-3
$$

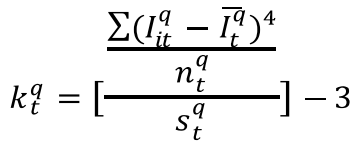

$$
\text { , siendo } p, q \subseteq J ; p \neq q
$$

\section{¿Cómo estimar la ideología de los individuos?}

Para medir la ideología de los individuos se utiliza el método propuesto en Poole (1998), el cual estima indirectamente la ideológica de los encuestados en función de la evaluación que realizan a un conjunto de actores políticos. Se asume que las personas tienen un grupo de creencias que explican sus opiniones políticas. Luego, se asume que un encuestado evalúa mejor a un actor político en la medida que se sienta más cercano a él en cuanto a dichas creencias (Converse 1964). Luego, se supone que dichas creencias son ideologías (Hinich y Munger1994).

Formalmente, se plantea que la evaluación hecha por un individuo $i$ sobre un tema $j, \mathrm{x}_{\mathrm{ij} j^{\prime}}$ está en función de su ideología, $I_{i^{\prime}}$ y un error estocástico, $e_{i}$ :

$$
x_{i j}=I_{i}+e_{i}
$$

Lo anterior también se puede representar a través del siguiente sistema de ecuaciones, considerando que en cada encuesta hay $m$ preguntas y $n$ encuestados:

$$
x=a^{*} I+e
$$

Luego, $x$ es una matriz de orden $n^{*} m, I$ es un vector $n^{*} 1$ y e es un vector de errores, en donde se asume que están distribuidos simétricamente en torno a cero. La ideología — variable latente que explica las opiniones en $x$ - corresponde a los valores singulares descompuestos para cada individuo $i$ en el caso que la matriz $x$ sea positiva y en que todos los individuos hayan respondido las $m$ preguntas. 
Una primera dificultad de este método es que comúnmente no todos los encuestados responden todas las preguntas. En este caso, Poole (1998) muestra que la matriz $x$ con celdas vacías aún puede estimarse a través de un proceso iterativo de maximización. Por lo tanto, a pesar de esta restricción, es posible estimar para cada individuo $i$ la variable latente $I_{i}$ utilizando el método mencionado.

Un segundo problema se refiere a que la ideología estimada $I_{k}$ no tiene una métrica intrínseca, lo cual dificulta la conmensurabilidad intertemporal. Este proceso de estimación indirecta extrae la posición relativa del individuo en función de su respuesta y las del resto de encuestados. Es decir, un valor específico de $I_{k}$ en una encuesta, no necesariamente significa lo mismo en otro sondeo. Por consiguiente, es necesario estandarizar la ideología estimada, tomando como referencia puntos fijos de la distribución para realizar un análisis entre 1990 y 2017. Después de ordenar a los encuestados de izquierda a derecha en función de $I_{k^{\prime}}$ se selecciona para cada encuesta - como referenciael percentil $21\left(p_{21}\right)$ y el percentil $80\left(p_{80}\right)$. Estos dos puntos son, respectivamente, el límite izquierdo del quintil de centro izquierda y el límite derecho del quintil de centro derecha. Posteriormente, en cada encuesta, la ideología estimada $I_{k}$ se transforma linealmente a una escala común entre -100 y 100, a través de la siguiente ecuación:

$$
\overline{I_{K}}=100-\left[\frac{200}{P_{80}-P_{21}}\right] \cdot b+I_{K} \cdot\left[\frac{200}{P_{80}-P_{21}}\right]
$$

\section{Datos}

Se utilizan los datos de la Encuesta de Opinión Pública del cEP. Desde el retorno a la democracia, este instrumento se ha aplicado de manera periódica, recopilando la opinión de los chilenos en diversos temas públicos. Este trabajo utiliza la siguiente pregunta de evaluación a actores políticos para estimar indirectamente la ideología de los encuestados:

"Ahora le voy a leer una lista de personas y quiero que me diga qué opina de cada una de ellas. Si usted no ha oído hablar de alguna de las personas, por favor, dígame que no la conoce. Usando las alternativas de esta tarjeta ¿cuál de estas frases describe mejor su opinión sobre cada persona?" (Centro de Estudios Públicos 2003).

Dado que las alternativas de respuesta son "muy positiva", "positiva", "ni positiva ni negativa", "negativa" y "muy negativa", se asume que los encuestados tendrán una opinión más positiva del actor político a medida que lo perciba más cercano ideológicamente. La validez de este supuesto se mantiene independiente de la aparición de eventos fortuitos que empeoren/ mejoren la evaluación transversal a los actores políticos. Esto descansa en el hecho que el proceso de estimación extrae la posición relativa del respondiente. Esto significa que, en el caso de una baja general de la evaluación a los políticos, 
un encuestado de centro, en vez de describir a un político de centro como "muy positivo", lo hará como "ni positivo ni negativo". Sin embargo, el encuestado de centro izquierda, en vez describirlo como "ni positivo ni negativo", lo hará como "negativo". De esta forma, independiente del empeoramiento generalizado de las evaluaciones hechas a los actores políticos, se mantendría el orden relativo de los respondientes al momento de evaluarlos.

Para cada encuesta se seleccionan a ocho actores políticos, siendo los criterios de selección (i) el número de apariciones durante el periodo de estudio y (ii) el número de evaluaciones recibidas en una encuesta, priorizando a los actores con mayor número de evaluaciones. En el caso que no apareciera el actor que se estaba evaluando, se reemplaza por otro con similitud política y que cumpla el criterio (ii). Por ejemplo, entre 1990 y 1994, se utilizan indistintamente a Augusto Pinochet, Hernán Buchi y a Arturo Alessandri como actor más a la derecha. En total, se evaluaron 488 actores políticos.

La ideología se calcula a través del comando basicspace del programa estadístico $R$. Se estima la ideología de los encuestados que evalúan a tres o más políticos. Sin embargo, se excluyen a quienes evalúan a todos los actores de la misma forma. Se realizaron 61 estimaciones durante el periodo 1990-2017, lo cual equivale al $92 \%$ del total de encuestas realizadas por el CEP en dicho periodo. Aquellas que no se consideran son: por no estar disponibles al momento de realizar esta investigación o las bases de datos o las preguntas utilizadas. De esta forma, se logra estimar la ideología de 80.999 individuos, equivalente al $88,1 \%$ del total de encuestados.

A continuación, se presenta evidencia que respalda la validez de la metodología y de los datos usados para estimar ideología. En primer lugar, la Figura 2 presenta la distribución ideológica de los encuestados y la posición estimada de los actores políticos evaluados en diciembre de 2006 y septiembre-octubre de 2013. Estos dos casos ilustran que el método posiciona a los políticos de izquierda a derecha de acuerdo a lo teóricamente esperado. En segundo lugar, la Figura 3 muestra la ideología promedio de los votantes de los principales candidatos presidenciales en las elecciones ocurridas durante el periodo en análisis. Los votantes se ordenan de izquierda a derecha, nuevamente de manera consistente con lo esperado. En consecuencia, se aprecia que la metodología de Poole (1998) y la serie del CEP estarían permitiendo rescatar la variable ideológica de los individuos.

Posteriormente, en el análisis de polarización entre-grupo se realiza lo siguiente. En cuanto a la posición política de los chilenos, se utiliza la pregunta de aprobación al gobierno de turno, clasificando como adherente a quienes aprueban al gobierno, como opositor a quienes lo rechazan y como neutro a los que ni aprueba ni rechazan. En cuanto al estrato socioeconómico, se agrupa al ABC1 y C2 como grupo alto, al C3 como grupo medio y como grupo bajo los segmentos D y E. En cuanto a lo generacional, se construyen tres categorías: los 
nacidos en 1955 o antes, los nacidos entre 1956 y 1980 y los que nacen en 1981 o después. La justificación de aquello radica en que los primeros son adultos (tenían 18 años o más) en el momento del quiebre democrático de 1973; los segundos no alcanzan a serlo en dicha fecha, pero sí lo son durante el régimen militar y en el periodo democrático en que Pinochet estuvo en el Ejército (hasta 1998); y los terceros, llegaron a ser adultos sin pasar por alguno de estos hechos.

Por último, el análisis de polarización ideológica se realiza a tres niveles. Primero en la opinión pública, que corresponde al total de individuos encuestados; segundo, en el electorado, que es la submuestra estimada de respondientes que vota en elecciones; y tercero, en el "no electorado", que es la submuestra de individuos que no vota. La ventaja de esto radica en un análisis más preciso de los segmentos objetivos de los políticos y más detallado de las tendencias identificadas a nivel de opinión pública. En 44 de las 61 encuestas (72\%), es posible construir submuestras equivalentes al "electorado" y "no electorado". De este modo, se analiza prácticamente la totalidad del periodo en estudio (1991-2017). La Tabla 1 presenta la descripción de los datos provenientes de las 61 encuestas.

Figura 2. Distribución ideológica de individuos y posición ideológica de actores políticos (diciembre 2006 y septiembre-octubre 2013)

a. Diciembre 2006

b. Septiembre-octubre 2013
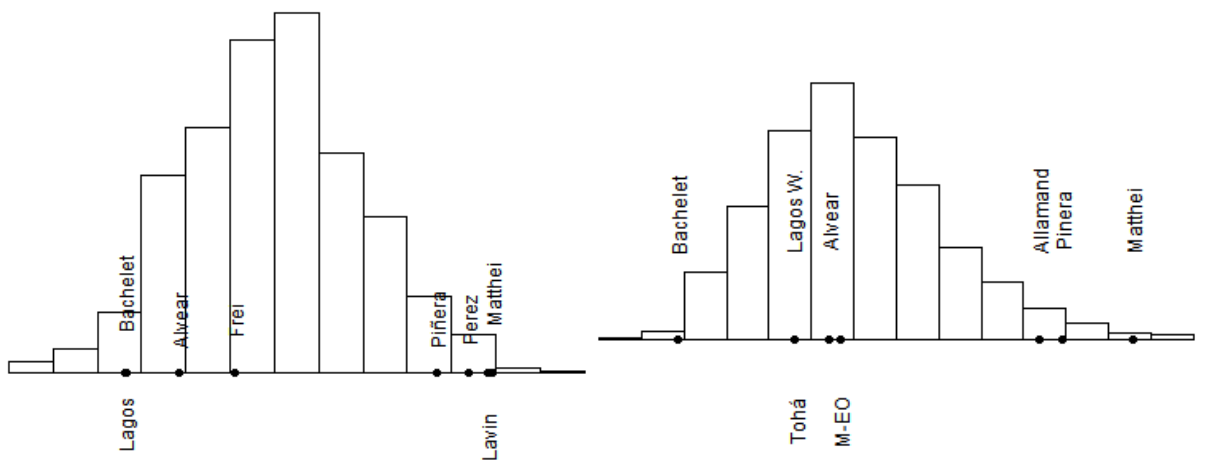

Fuente: elaboración propia 
Figura 3. Media ideológica de votantes de los principales candidatos presidenciales (1990-2017)

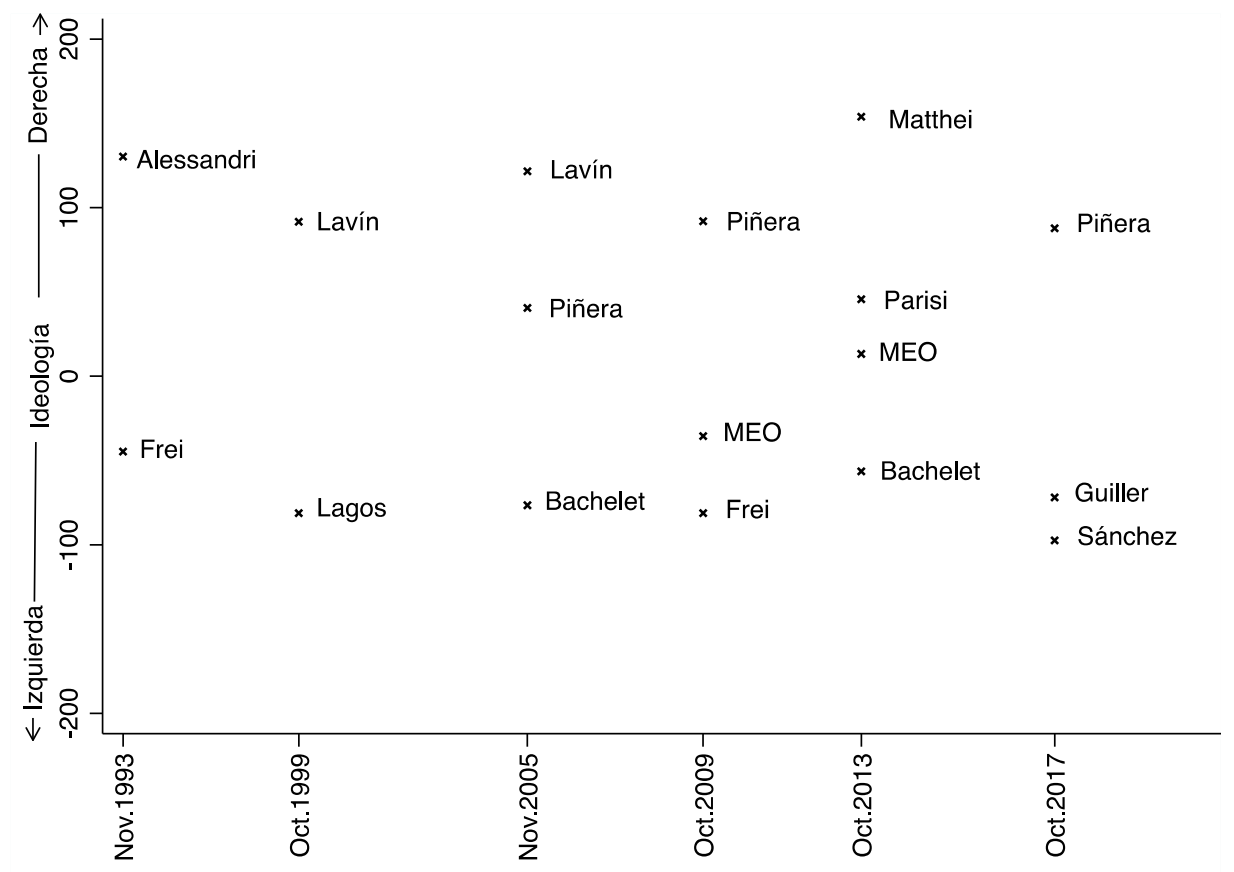

Fuente: elaboración propia

Tabla 1. Descripción de los datos de las 61 encuestas (1990-2017)

\begin{tabular}{|c|c|c|c|c|c|c|}
\hline Variable & Obs. & $\%$ & Media & $\begin{array}{c}\text { Desv. } \\
\text { Estándar }\end{array}$ & Mín. & Max. \\
\hline Ideología & 80,999 & $(-)$ & 0.72 & 117.44 & -429.97 & 472.56 \\
\hline \multicolumn{7}{|l|}{ Electorado } \\
\hline Electorado & 43,377 & $76.8 \%$ & $(-)$ & $(-)$ & $(-)$ & $(-)$ \\
\hline No Electorado & 13,090 & $23.2 \%$ & $(-)$ & $(-)$ & $(-)$ & $(-)$ \\
\hline Total & 56,467 & & & & & \\
\hline
\end{tabular}

Aprobación al gobierno de turno

$\begin{array}{lllllll}\text { Adherente } & 36,706 & 46.8 \% & (-) & (-) & (-) & (-) \\ \text { Opositor } & 26,355 & 33.6 \% & (-) & (-) & (-) & (-) \\ \text { Neutro } & 15,347 & 19.6 \% & (-) & (-) & (-) & (-) \\ \text { Total } & \mathbf{7 8 , 4 0 8} & & & & & \end{array}$




\begin{tabular}{ccccccc}
\hline Variable & Obs. & $\%$ & Media & $\begin{array}{c}\text { Desv. } \\
\text { Estándar }\end{array}$ & Mín. & Max. \\
\hline $\begin{array}{c}\text { Nivel socioeconómico } \\
\text { Alto }\end{array}$ & 11,802 & $14.6 \%$ & $(-)$ & $(-)$ & $(-)$ & $(-)$ \\
Medio & 32,364 & $40.0 \%$ & $(-)$ & $(-)$ & $(-)$ & $(-)$ \\
Bajo & 36,644 & $45.3 \%$ & $(-)$ & $(-)$ & $(-)$ & $(-)$ \\
Total & $\mathbf{8 0 , 8 1 0}$ & & & & & \\
\hline Generación & & & & & & \\
1955 o antes & 23,802 & $36.4 \%$ & $(-)$ & $(-)$ & $(-)$ & $(-)$ \\
1956-1980 & 32,144 & $49.2 \%$ & $(-)$ & $(-)$ & $(-)$ & $(-)$ \\
1981 o después & 9,359 & $14.3 \%$ & $(-)$ & $(-)$ & $(-)$ & $(-)$ \\
\hline Total & $\mathbf{6 5 , 3 0 5}$ & & & & &
\end{tabular}

(a) Corresponde al total de individuos con ideología estimada pertenecientes a las 44 encuestas del total de 61 en que se logró identificar votantes.

Fuente: elaboración propia

\section{RESULTADOS}

La primera parte de esta sección discute sobre los resultados relativos a la polarización de la población. La segunda fracción lo hará respecto a la polarización entre grupos, agrupando a los chilenos en función de la adherencia al gobierno de turno, nivel socioeconómico y generación. Se estudió la polarización entre grupos tomando en cuenta el género y la residencia de los encuestados. Sin embargo, los resultados no se presentan al no mostrar rasgos de polarización.

Se utilizan las regresiones locales ponderadas robustas propuestas por Cleveland (1979) para presentar los resultados de manera más clara. Además, se estima una regresión lineal entre cada indicador en estudio (diferencia media, varianza y kurtosis) y una variable tiempo, con el objetivo de identificar patrones temporales. En el caso que el coeficiente estimado relativo a la variable tiempo sea significativo, se sostiene que el indicador presenta un patrón temporal creciente o decreciente, dependiendo del signo de la estimación. Estos resultados se presentan en los Anexos.

\section{Polarización de la población}

La Figura 4 muestra la evolución de la varianza y kurtosis de la distribución ideológica de los chilenos.

A nivel de opinión pública, la dispersión ideológica se mantiene constante, mientras que la modalidad aumenta entre 1990 y 2008. A partir de 2009, la kurtosis decrece. A nivel del electorado hay un comportamiento similar: una dispersión estable y una modalidad que aumenta hasta 2008, pero que luego cae. En ambos casos, la caída en la kurtosis podría estar revelando una 
incipiente polarización de la población, toda vez que se complemente con una mayor dispersión. Además, los votantes muestran - respecto a la opinión pública - tanto una mayor varianza como una menor kurtosis, lo que indicaría un electorado más polarizado. En contraste, el "no electorado" tiene un mayor consenso poblacional, al tener una menor dispersión y mayor kurtosis respecto a los casos anteriores.

En suma, no hay evidencia para sostener la existencia de polarización de la población entre 1990 y 2017. La dispersión se mantiene relativamente constante y la modalidad ideológica aumenta. Pese aquello, la caída en la modalidad observada desde 2009 podría evidenciar, a futuro, una polarización de la población entre los chilenos, en la medida que aumente la dispersión.

\section{Polarización entre grupos}

Como se señaló, el análisis de la polarización entre grupos considera dos dimensiones: la heterogeneidad entre-grupo y homogeneidad intra-grupo. Para el análisis a nivel socioeconómico y generacional, se exponen la evolución de las medias ideológicas y de las kurtosis. En cambio, a nivel de adherencia al gobierno de turno, se presenta la diferencia de media de los grupos, con el fin de neutralizar los cambios de signos que implica la alternancia de gobierno.

Se había planteado que el cambio, desde un contexto político atrincherado propio del régimen de Pinochet- hacia una época de acuerdos, representó uno de los cambios más relevantes que vivieron los chilenos durante las últimas tres décadas. Al respecto, la Figura 5 muestra evidencia de que ellos se polarizaron en cuanto a sus posiciones políticas. Pese a un breve momento de acercamiento, prácticamente en la totalidad de la década de 1990 y en los primeros años del siglo XXI, adherentes y opositores se fueron distanciando ideológicamente, proceso que duró hasta 2008 a nivel de "opinión pública" y hasta 2014 en tanto a "electorado" y "no electorado". En cuanto a la magnitud de este alejamiento, se ve una mayor polarización entre los "votantes" (valores en torno a 150) que entre la "opinión pública" (valores bajo 150) y que entre los "no votantes" (valores en torno a 100). Resalta el hecho que entre los "votantes", el proceso de distanciamiento se mantuvo hasta 2017, a diferencia de la "opinión pública" en que se atenúa a partir de 2014. 
Figura 4. Evolución de varianza y kurtosis ideológica, en opinión pública, electorado y no electorado (1990-2017)
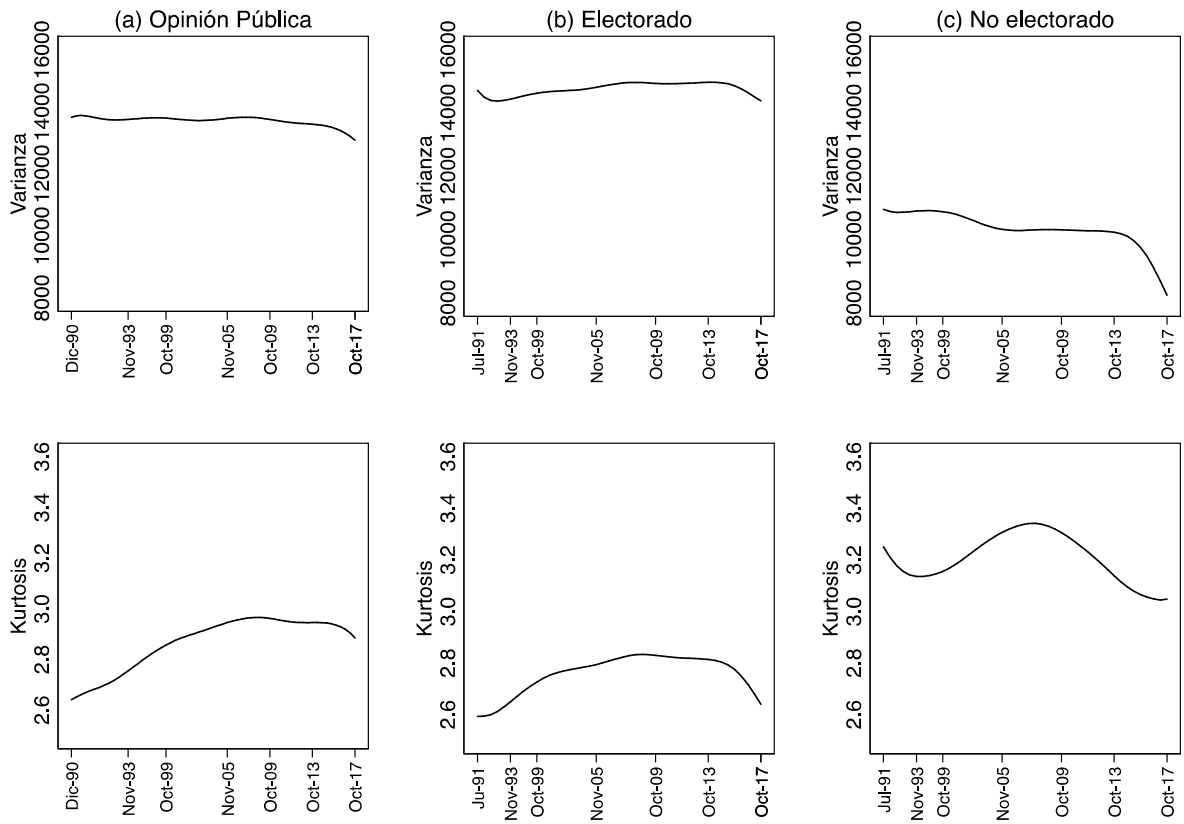

Fuente: elaboración propia

Las posiciones políticas de los chilenos no solo se volvieron más heterogéneas unas con otras, sino que más homogéneas internamente. La Figura 5 revela que, tanto adherentes como opositores, se tornaron más cohesionados al interior de cada grupo. Este hecho ocurre en la "opinión pública" y en los "votantes", no así en los "no votantes". Ahora bien, a fines de 2014, la homogeneidad intra-grupo de los opositores al gobierno de turno empieza decrecer, lo cual condice con el proceso de división de la coalición de centro izquierda.

A pesar de que la política chilena pasó desde un contexto de extrema beligerancia hacia uno más colaborativo en la transición, los chilenos se volvieron más polarizados en cuanto sus posiciones políticas entre 1990 y 2017. Opositores y adherentes tomaron distancia uno con otros y, además, se volvieron internamente más cohesionados. 
Figura 5. Evolución de media y kurtosis ideológica, por adherencia al gobierno de turno, en opinión pública, electorado y no electorado (1990-2017)

(a) Opinión Pública
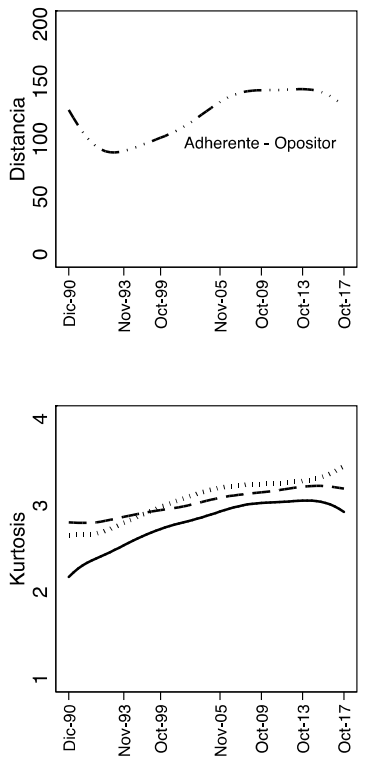

Adherente

Neutro

Fuente: elaboración propia (b) Electorado
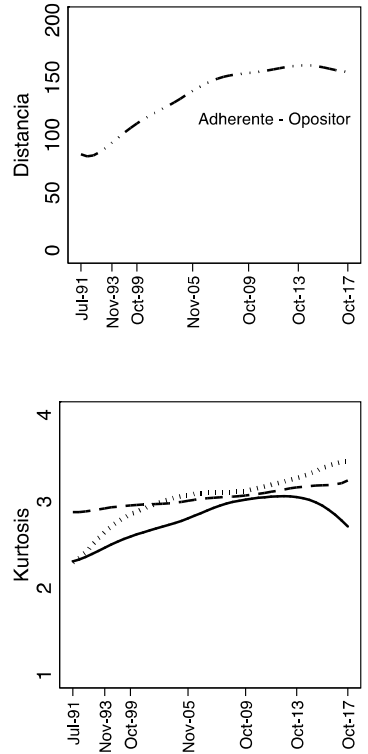

Opositor (c) No electorado
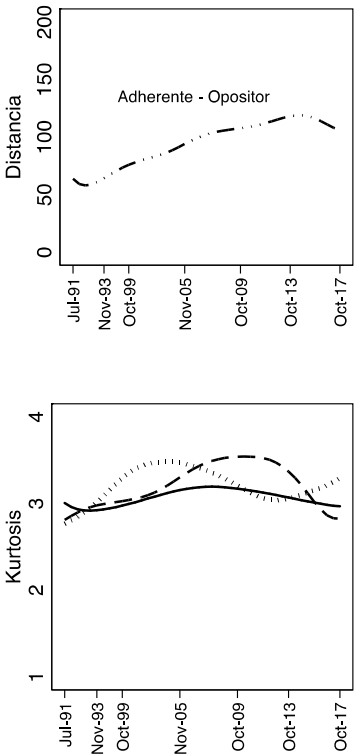

La sociedad chilena no solo vivió transformaciones políticas, sino que también materiales, las cuales condicen con una polarización ideológica a nivel socioeconómico, como muestra la Figura 6. En contraste a lo visto, en la última década del siglo XX existió un acercamiento ideológico, principalmente entre los grupos alto y bajo. No obstante, a partir de 2005, ambos estratos empezaron a distanciarse, alcanzando una diferencia inclusive mayor respecto a la existente al inicio de los 90'. A nivel de votantes, este fenómeno se acentúa.

Además, los grupos socioeconómicos bajos y medios se vuelven ideológicamente más homogéneos. No hay evidencia para sostener lo mismo en el grupo alto. Entre los "votantes" solo el grupo bajo se tornó más homogéneo, mientras que entre los "no votantes" este proceso de cohesión en el grupo bajo se invierte desde 2008.

En suma, hay una mayor polarización ideológica en términos socioeconómicos: el grupo alto y bajo están más distantes y, además, este último es más homogéneo. 
Figura 6. Evolución de media y kurtosis ideológica, por nivel socioeconómico, en opinión pública, electorado y no electorado (1990-2017)

(a) Opinión Pública
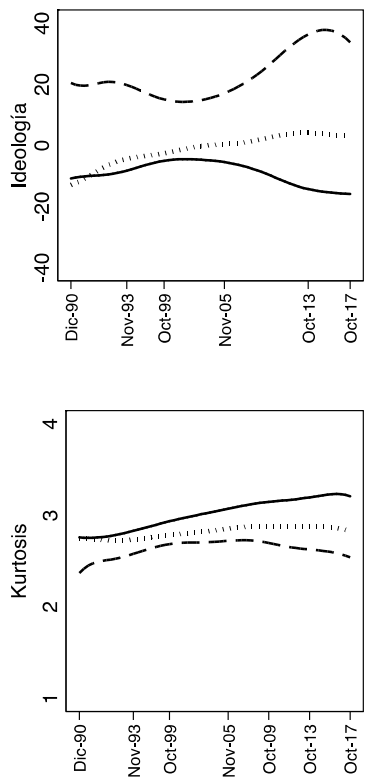

(b) Electorado
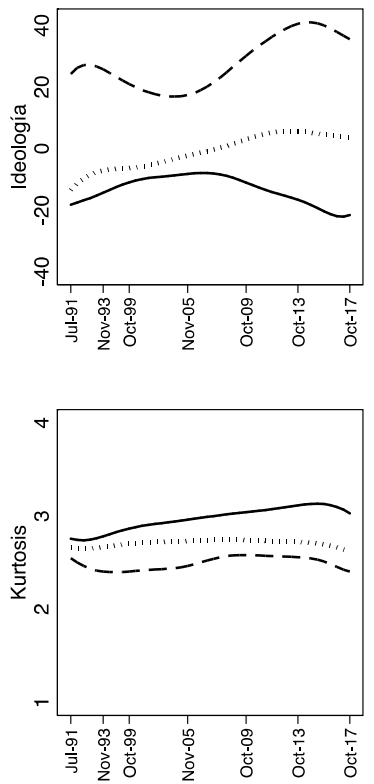

(c) No electorado
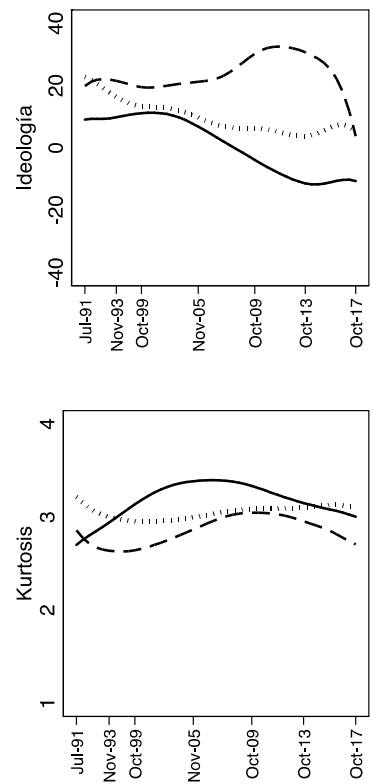

Alto - - - Medio $111 \ldots 1 \cdots 1$ Bajo

Fuente: elaboración propia

Las transformaciones políticas y económicas generaron una brecha generacional, puesto que quienes nacieron con posterioridad no vivieron contextos políticos tan álgidos como las generaciones mayores y además se beneficiaron directamente de los cambios del crecimiento. Por ello se extrapola la relevancia de indagar sobre la polarización ideológica a nivel generacional.

La Figura 7 muestra que las tres generaciones se mantuvieron cercanas en gran parte del tiempo. Sin embargo, 2013 es un punto de inflexión: se inicia un incipiente distanciamiento entre los que eran adultos en 1973 y las otras dos generaciones, tendiendo a la izquierda y a la derecha, respectivamente. Esto se ve más claro entre los "votantes", en quienes empezaron a crecer las diferencias entre la generación mayor y menor a partir del 2011. Entre los "no votantes", los datos muestran una tendencia distinta: las tres generaciones se mueven hacia la izquierda. Por otra parte, la generación más joven se cohesionó internamente hasta el periodo 2005-2006, tanto a nivel de "opinión pública" como "electorado". La generación que vivió el golpe militar ha mantenido una kurtosis estable en el tiempo, mientras que la generación nacida en el periodo 1956-1980, si bien tuvo una kurtosis estable, entre 2014 y 2015 empezó a decrecer. Esto último ocurre tanto en la "opinión pública" como "electorado". 
Figura 7. Evolución de media y kurtosis ideológica, por generación, en opinión pública, electorado y no electorado (1990-2017)

(a) Opinión Pública
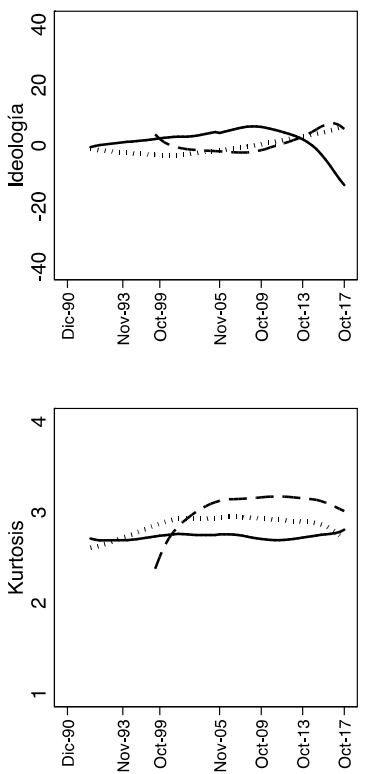

(b) Electorado
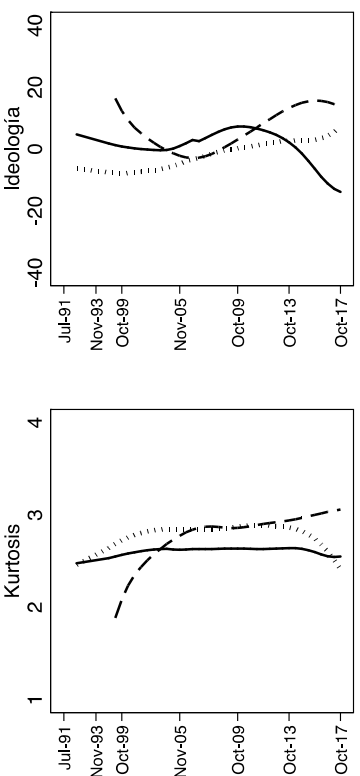

(c) No electorado
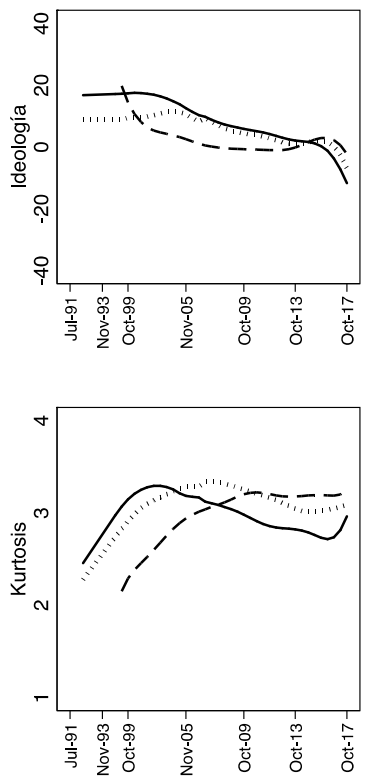

Desde 1981 - - 1956-1980,

Hasta 1955

Fuente: elaboración propia

\section{CONCLUSIÓN}

Este artículo estudió los cambios ideológicos que experimentaron los chilenos durante el periodo 1990 a 2017, entregando evidencia de polarización ideológica a nivel político, socioeconómico y generacional.

En primer lugar, los datos muestran que ha existido una creciente polarización política entre ciudadanos de Chile que se manifestaron adherentes y opositores a los gobiernos de turno. Desde 1993 empezaron a vivir un proceso de distanciamiento que persistió hasta 2008. Y no solo eso, ambos grupos se volvieron internamente más homogéneos. En suma, los chilenos —en cuanto a sus posiciones políticas - se distanciaron unos de otros y se cohesionaron al interior de sus respectivos grupos. Estos resultados son llamativos, toda vez que los niveles de polarización política vistos en la actualidad son inclusive mayores al inicio de la década de 1990, época en la cual aún existía un grado de estridencia respecto la figura de Pinochet.

En segundo lugar, el artículo ofrece evidencia de polarización ideológica entre el grupo socioeconómico alto y bajo. Se observó que, desde 2005, la brecha ideológica entre ambos aumentó en el tiempo. Asimismo, entre 1990 y 2017, el grupo bajo se volvió internamente más homogéneo. Estos resultados son 
llamativos ya que las transformaciones materiales que vivieron los chilenos condicen con una mayor polarización socioeconómica en el tiempo.

Por último, se presentaron resultados consistentes con una incipiente polarización ideológica generacional. En particular, entre la que vivió el golpe militar del 1973 y la que llegó a la adultez sin Pinochet en la esfera política. El punto de inflexión a nivel de "opinión pública" es el año 2013, mientras que entre los "votantes" corresponde a 2011. El surgimiento —a mediados de la primera década de los 2000 - de la facción "autoflagelante" en la Concertación podría haber traído como consecuencia que aquellas personas que vivieron el golpe militar, hayan extremado sus posiciones ideológicas frente a la nueva retórica. En contraste, la generación "nacida en democracia" no era del todo susceptible a ella. Otra explicación podría venir desde la evidencia entregada por Sapelli (2014), en cuanto a que altos niveles de desigualdad en la generación nacida en la primera mitad del siglo XX persisten, en contraste a las nuevas generaciones en las que este tipo de inequidad es menor.

Hace al menos diez años se empezaron a ver —en la discusión públicadistintas posiciones respecto a la presencia o no de polarización en la población chilena. Esta investigación entrega evidencia de que, efectivamente, existe este fenómeno en Chile, el cual no se circunscribe exclusivamente ni al último gobierno de Bachelet (2014-2018) ni a las movilizaciones sociales, sino que es un proceso que viene - en término de posiciones políticas - desde los primeros años del retorno a la democracia. Luego, con unos años de rezagos a las primeras manifestaciones sociales, empezó a surgir socioeconómicamente y, recién en los últimos años, se ha revelado en términos generacionales. Estos hallazgos revelan que los consensos tras el retorno a la democracia son frágiles frente una población que está ideológicamente más polarizada.

\section{REFERENCIAS}

Abramowitz, Alan y Kyle Saunders. 2006. "Exploring the Bases of Partisanship in the American Electorate: Social Identity vs. Ideology". Political Research Quarterly 59 (2): 175-187.

Abramowitz, Alan y Kyle Saunders. 2008. "Is Polarization a Myth?". Journal of Politics 70 (2): 542-555.

Abramowitz, Alan. 2010. The Disappearing Center: Engaged Citizens, Polarization, and American Democracy. New Haven: Yale University Press.

Agencia oRBE. 2007, 27 de octubre. "Walker llama a retornar a la política de consenso". El Mercurio On-Line (EмOL). Recuperado el 8 de septiembre de 2018 de http:/ /www.emol. com/noticias/nacional/2007/10/27/279959/walker-llama-a-retornar-a-la-politicade-los-consensos.html

Agencia UPI. 2011, 23 de septiembre. "Accorsi y Tarud llamaron a formar mesas de diálogo para mejorar el clima político". ADN Radio. Recuperado el 8 de septiembre del 2018 de http:/ / www.adnradio.cl/noticias/politica/accorsi-y-tarud-llamaron-a-formar-mesas-de-dialogo-para-mejorar-clima-politico/20111023/nota/1566552.aspx

Baldassarri, Delia y Andrew Gelman. 2008. "Partisans without Constraint: Political Polarization and Trends in American Public Opinion". American Journal of Sociology 114 (1): 408-446. 
Baldassarri, Delia y Peter Bearman. 2007. "Dynamics of Political Polarization". American Sociological Review 72 (1): 784-811.

Bonilla, Claudio. 2002. "A Micro Application of the Spatial Theory of Voting". Revista de Ciencia Política 21 (2): 3-16.

Brady, David y Hahrie Han. 2004. "An Extended Historical View of Congressional Party Polarization". Ponencia presentada en Princeton University, 2 de diciembre.

Çarkoğlu, Ali y Melvin Hinich. (2006). "A Spatial Analysis of Turkish Party Preferences". Electoral Studies 25 (2): 369-392.

Centro de Estudios Públicos (CEP). 2003. Estudio nacional de opinión pública $n^{\circ} 17$ (Tercera serie, junio-julio 2003, CEP0045-v1). Santiago: Centro de Estudios Públicos.

Cleveland, William S. 1979. "Robust Locally Weighted Regression and Smoothing Scatterplots". Journal of the American Statistical Association 74 (1): 829-836.

Constable, Pamela y Arturo Valenzuela. 1993. A Nation of Enemies: Chile Under Pinochet. New York: Norton.

Converse, Philip. 1964. "The Nature of Belief Systems in Mass Publics". En Ideology and Discontent, editado por David Apter. New York: Free Press, 206-261.

Cox, Gary. 1990. "Centripetal and Centrifugal Incentives in Electoral Systems". American Journal of Political Science 34 (4): 903-935.

Dalton, Russell J. 2008. "The Quantity and the Quality of Party Systems". Comparative Politics Studies 41 (7): 899-920.

Di Maggio, Paul, John Evans y Bethany Bryson. 1996. "Have Americans' Social Attitudes Become More Polarized?". American Journal of Sociology 102 (1): 690-755.

Dow, Jay K. 1998. "A Spatial Analysis of Candidate Competition in Dual Member Districts: The 1989 Chilean Senatorial Elections". Public Choice 97 (1): 451-474.

Downey, Dennis y Matt Huffman. 2001. "Attitudinal Polarization and Trimodal Distributions: Measurement Problems and Theoretical Implications". Social Science Quarterly 82 (3): 494-505.

Downs, Anthony. 1957. "An Economic Theory of Political Action in a Democracy". Journal of Political Economy 65 (2): 135-150.

El Mercurio. 2014, 16 de mayo. "La disputa por la clase media". El Mercurio, A3. Recuperado el 9 de abril de 2019 de http://www.elmercurio.com/blogs/2014/05/16/21875/Ladisputa-por-la-clase-media.aspx

Enelow, James y Melvin Hinich. 1984. The Spatial Theory of Voting: An Introduction. New York: Cambridge University Press.

Esteban, Joan-Maria y Debray Ray. 1994. "On the Measurement of Polarization". Econometrica 62 (1): 819-851.

Evans, John. 2003. "Have Americans' Attitudes Become More Polarized? An Update". Social Science Quarterly 84 (1): 71-90.

Fiorina, Morris y Samuel Abrams. 2008. "Political Polarization in the American Public". Annual Review of Political Science 11 (1): 563-588.

Fiorina, Morris, Samuel Abrams y Jeremy Pope. 2005. Culture War? The Myth of a Polarized America. New York: Longman.

Fondo Monetario Internacional. 2017. World Economic Outlook. Seeking Sustainable Growth: Short-Term Recovery, Long-Term Challenges. Washington: International Monetary Fund.

García, Fernanda. 2018, 25 de marzo. "Nos polarizamos mucho con el gobierno anterior". El Mercurio de Valparaíso, D 6-7. Recuperado el 8 de septiembre de 2018 de http:/ /www. mercuriovalpo.cl/impresa/2018/03/25/full/cuerpo-reportajes/6/

Gelman, Andrew. 2008. Red State, Blue State, Rich State, Poor State: Why Americans Vote the Way they do. New Jersey: Princeton University Press.

Hegselmann, Rainer y Ulrich Krause. 2002. “Opinion Dynamics and Bounded Confidence: Models, Analysis, and Simulation". Journal of Artificial Sciences and Social Simulation 5 (1): 1-3.

Hetherington, Marc. 2008. "Turned off or Turned on? How Polarization Affects Political Engagement". En Red and Blue Nation? Consequences and Correction of America's Polarized 
Politics, Vol. 2, editado por Pietro Nivola y David Brady. Washington: Brookings Institution Press,1-33.

Hinich, Melvin. 1977. "Equilibrium in Spatial Voting: The Median Voter Result is an Artifact". American Journal of Political Science 25 (2): 323-341.

Hinich, Melvin y Michael Munger. 1994. Ideology and the Theory of Political Choice. Ann Arbor: The University of Michigan Press.

Layman, Geoffrey C., Thomas Carsey y Juliana Menasce Horowitz. 2006. "Party Polarization in American Politics: Characteristics, Causes, and Consequences". Annual Review of Political Science 9 (1): 83-110.

Lee, Jae M. 2015. “Assesing Mass Opinion Polarization in the US Using Relative Distribution Method". Social Indicator Research 124 (1): 571-598.

León, Luis Eduardo. 2013. "La teoría espacial del voto. Una propuesta de aplicación en Latinoamérica". Revista Mexicana de Análisis Político y Administración Pública 2 (2): 57-79.

Levendusky, M. 2009. The Partisan Sort. How Liberals Became Democrats and Conservatives Became Republicans. Chicago: University of Chicago Press.

Magar, Eric, Marc R. Rosenblum y David J. Samuels. 1998. "On the Absence of Centripetal Incentives in Double-Member Districts: The Case of Chile". Comparative Political Studies 31 (6): 714-739.

McCarty, Nolan, Keith T. Poole y Howard Rosenthal. 2006. Polarized America: The Dance of Ideology and Unequal Riches. Cambridge: MIT Press.

Morales, Mauricio. 2017. "Polarización y participación: la presidencial 2017". Radio T13. Recuperado el 8 de septiembre del 2018 de http://www.t13.cl/blog/columnas-mauricio-morales/polarizacion-y-participacion-presidencial-2017

Poole, Keith y Howard Rosenthal. 1984. "The Polarization of American Politics". The Journal of Politics 46 (1): 1061-1079.

Poole, Keith y Howard Rosenthal. 1997. Congress: A Political Economic History of Roll Call Voting. New York: Oxford University Press.

Poole, Keith. 1998. "Recovering a Basic Space from a Set of Issue Scales". American Journal of Political Science 42 (1) :954-993.

Rosenthal, Howard y Erik Voeten. 2004. "Analyzing Roll Calls with Perfect Spatial Voting: France 1946-1958". American Journal of Political Science 48 (3): 620-632.

Saiegh, Sebastián. 2014. "Partisanship, Ideology, and Representation in Latin America". IDB Working Paper Series (N ${ }^{\circ}$ IDB-WP-533). Washington: Inter-American Development Bank.

Sapelli, Claudio. 2014. “Desigualdad, movilidad, pobreza. Necesidad de una política social diferente". Estudios Públicos 134 (1): 58-84.

Sartori, Giovanni. 2005. Parties and Party Systems: A Framework for Analysis. Colchester: ECPR Press.

Valenzuela, Paula y David Muñoz. 2017, 17 de septiembre. "Harald Beyer, director del Centro de Estudios Públicos (CEP): Las derechas modernas apelan a la igualdad de derechos". La Tercera. Recuperado el 8 de septiembre del 2018 de http://www2.latercera.com/ noticia/harald-beyer-director-del-centro-estudios-publicos-cep-las-derechas-modernas-apelan-la-igualdad-derechos/

Recibido: 17 de agosto de 2017 Aceptado: 11 de octubre de 2018 
Jaime Lindh es magíster en Economía y Políticas Públicas por la Universidad Adolfo Ibáñez (Chile). Se desempeña como investigador en el centro de estudios IdeaPaís. Correo electrónico: jaime. lindh@ideapais.cl

Jorge Fábrega es doctor en Políticas Públicas por la Universidad de Chicago (EE. UU.). Dirige el Doctorado en Ciencias de la Complejidad Social en la Universidad del Desarrollo (Chile). Correo electrónico: jfabrega@udd.cl

Jorge González es doctor en Gobierno por la Universidad de Texas en Austin (EE. UU.). Es académico de la Escuela de Gobierno en la Universidad Adolfo Ibáñez, Campus Viña del Mar (Chile). Correo electrónico: jorge.gonzalez@uai.cl 


\section{ANEXOS}

Tabla 2. Varianza y kurtosis de la distribución ideológica, opinión pública, electorado y no electorado. Regresión entre indicador y tiempo

\begin{tabular}{lrrrrrr}
\hline & \multicolumn{3}{c}{ Varianza } & \multicolumn{3}{c}{ Kurtosis } \\
\hline & $\begin{array}{c}\text { Opinión } \\
\text { pública }\end{array}$ & Electorado & $\begin{array}{c}\text { No } \\
\text { electorado }\end{array}$ & $\begin{array}{l}\text { Opinión } \\
\text { pública }\end{array}$ & Electorado & $\begin{array}{c}\text { No } \\
\text { electorado }\end{array}$ \\
\hline Tiempo & $-3,9667$ & 11,7092 & $-29,9892$ & 0,0052 & 0,0037 & $-0,0011$ \\
& $(7,5271)$ & $(16,3987)$ & $(25,8337)$ & $(0,0016)^{* * *}$ & $(0,0029)$ & $(0,0052)$ \\
Constante & $13,841,54$ & $14,537,55$ & $11,102,34$ & 2,7145 & 2,6902 & 3,2342 \\
& $(268,3510)^{* * *}$ & $(423,6778)^{* * *}$ & $(667,4408)^{* * *}$ & $(0,0553)^{* * *}$ & $(0,0744))^{* * *}$ & $(0,1345)^{* * *}$ \\
\hline$R^{2}$ & 0 & 0,01 & 0,03 & 0,16 & 0,04 & 0 \\
$\mathrm{~N}$ & 61 & 44 & 44 & 61 & 44 & 44 \\
\hline
\end{tabular}

Error estándar en paréntesis ${ }^{*} p<0,1 ;{ }^{* *} p<0,05 ;{ }^{* *} p<0,01$

Fuente: Elaboración propia

Tabla 3. Heterogeneidad entre-grupo de adherencia, opinión pública, electorado y no electorado, regresión entre diferencia absoluta y tiempo

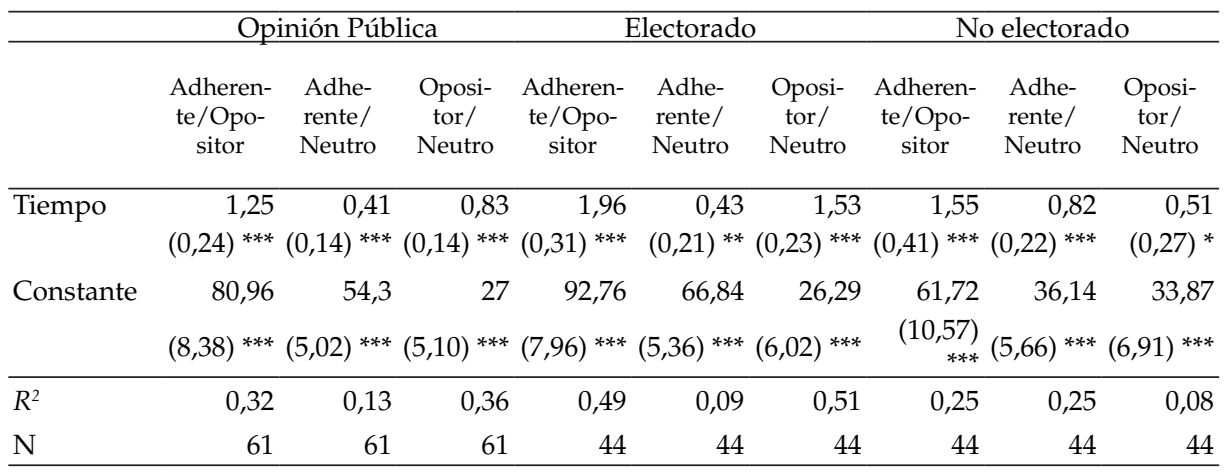

Error estándar en paréntesis ${ }^{*} p<0,1 ;{ }^{* *} p<0,05 ;{ }^{* * *} p<0,01$

Fuente: Elaboración propia 


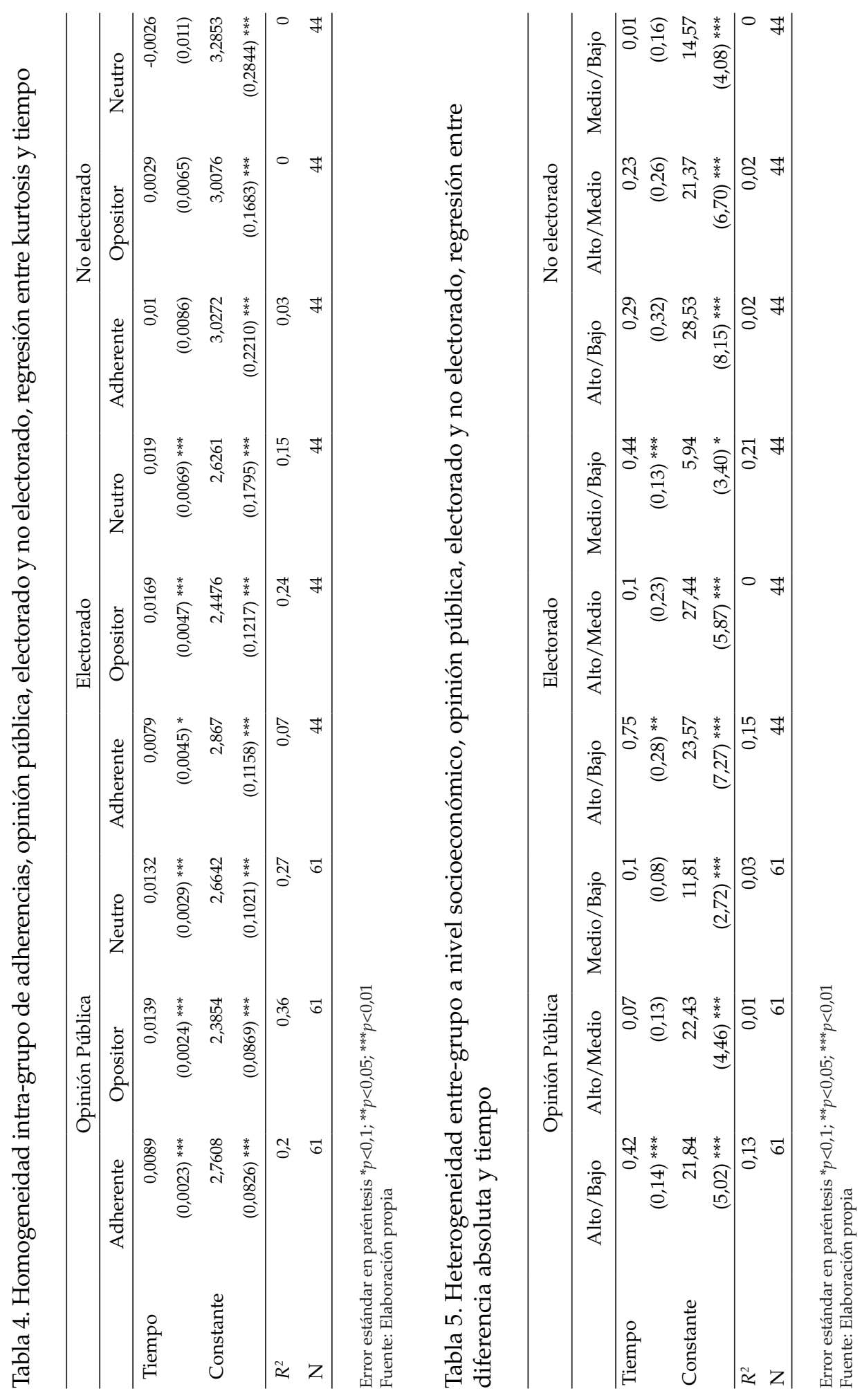




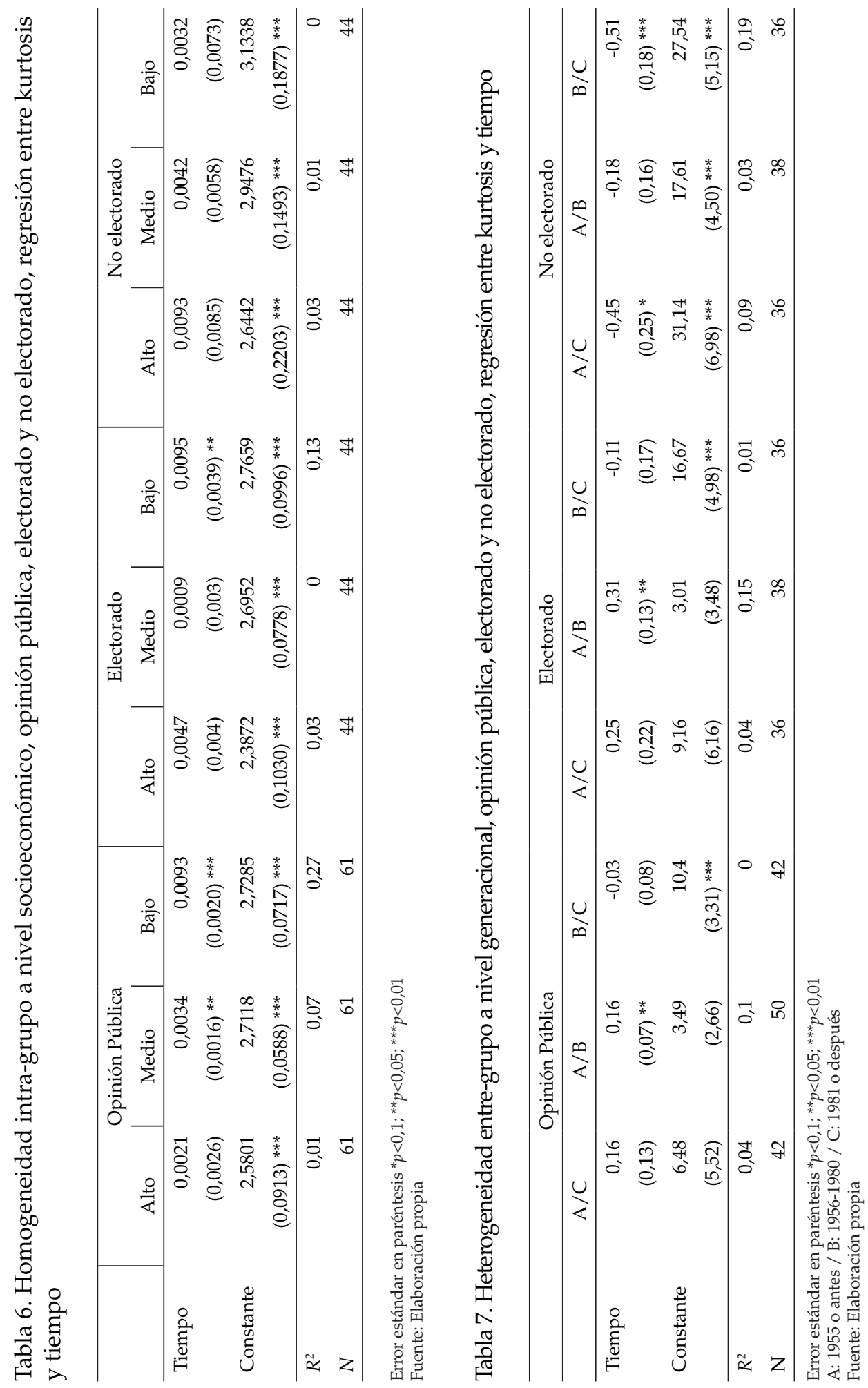




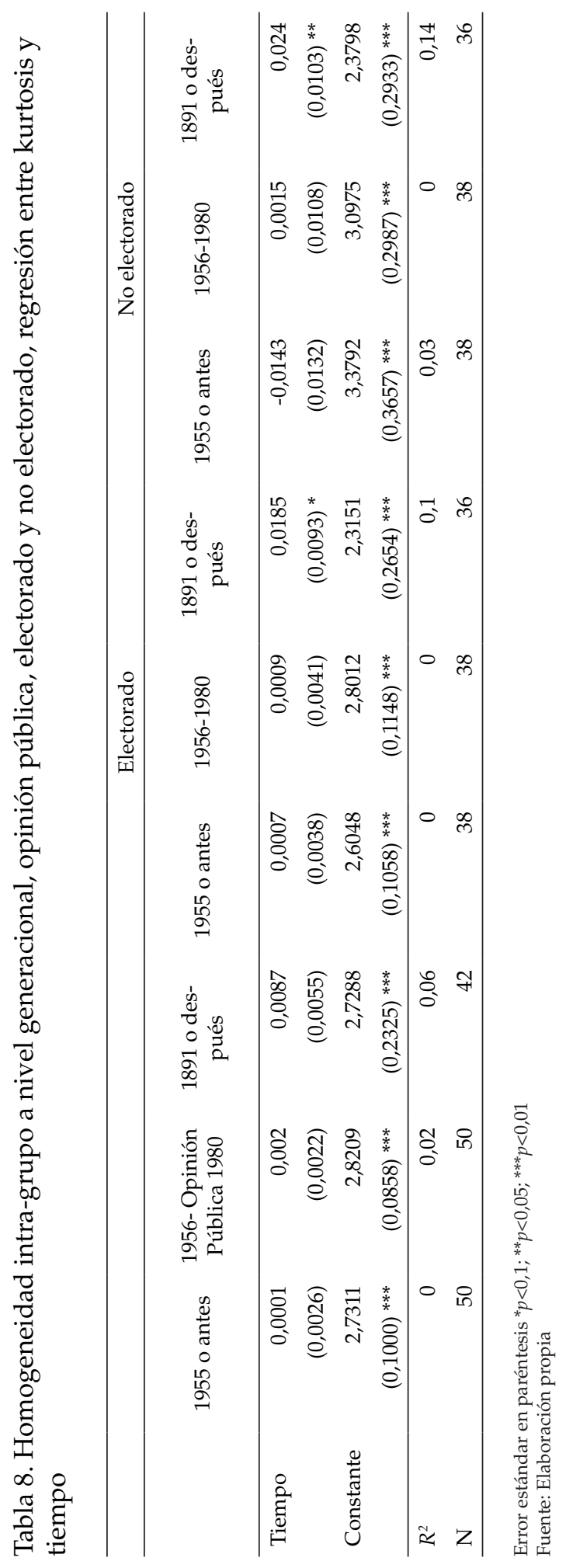

